\title{
ATTRACTORS FOR SEMILINEAR PARABOLIC PROBLEMS WITH DIRICHLET BOUNDARY CONDITIONS IN VARYING DOMAINS
}

\author{
Emerson A. M. de Abreu* Alexandre N. Carvalho ${ }^{\dagger}$
}

\begin{abstract}
Under fairly general conditions one can prove that the asymptotic dynamics of dissipative semi-linear autonomous parabolic problems is upper semi-continuous with respect to perturbations in the model. In this paper we prove, for such problems, that the asymptotic dynamics behaves continuously (upper and lower semi-continuously) with respect to perturbations of the spatial domain. We prove the continuity with respect to domain perturbations of the set of stationary states and use this, together with the continuity of eigenvalues and eigenfunctions of an elliptic operator to obtain the continuity of the local unstable manifolds of equilibria and consequently the continuity of attractors.
\end{abstract}

\section{Introduction}

Let $\epsilon_{0}$ be a positive number and $\Omega_{\epsilon} \subset \mathbb{R}^{N}, N \leq 4,0 \leq \epsilon \leq \epsilon_{0}$ be a family of bounded smooth domains. Also assume that, for any $K \subset \subset \Omega_{0}$ and $U \supset \Omega_{0}$, $U \subset \mathbb{R}^{N}$ open, there exists $\epsilon_{K, U}>0$ such that $K \subset \Omega_{\epsilon} \subset \bar{\Omega}_{\epsilon} \subset U, 0 \leq \epsilon \leq \epsilon_{K, U}$. We say that $\Omega_{\epsilon}, \epsilon>0$ is a perturbation of $\Omega_{0}$. Our aim is to study the continuity

Key words and phrases. parabolic equations, attractors, lower semi-continuity 1991 Mathematics Subject Classification. Primary 35B40; Secondary 35K55, 65P05

*Partially supported by FAPEMIG e: CEX-00102/01.

${ }^{\dagger}$ Partially supported by grant \# 300.889/92-5 CNPq 
of the asymptotic dynamics of parabolic problems of the form

$$
\begin{array}{ll}
u_{t}=\Delta u+f(u), & x \in \Omega_{\epsilon} \\
u=0, & x \in \partial \Omega_{\epsilon} \\
u(0)=u_{0} \in H_{0}^{1}\left(\Omega_{\epsilon}\right) &
\end{array}
$$

where $f: \mathbb{R} \rightarrow \mathbb{R}$ is a $C^{2}(\mathbb{R})$ function satisfying the growth condition

$$
\begin{gathered}
\left|f^{\prime}(u)\right| \leq c\left(|u|^{\frac{4}{N-2}}+1\right), \quad \text { if } \quad N=3,4, \text { or } \\
\lim _{|u| \rightarrow \infty} \frac{\left|f^{\prime}(u)\right|}{e^{\theta|u|^{2}}}=0, \forall \theta>0, \quad \text { if } \quad N=2
\end{gathered}
$$

(no growth condition is needed for $N=1$ ) and the dissipativeness condition

$$
\limsup _{|u| \rightarrow \infty} \frac{f(u)}{u} \leq 0 .
$$

The condition (1.2) ensures that the problem (1.1) is locally well posed; that is, for each $u_{0} \in H^{1}\left(\Omega_{\epsilon}\right)$ the problem (1.1) has a unique $\eta$-regular solution which depends continuously (in $H^{1}\left(\Omega_{\epsilon}\right)$ ) on the initial data $u_{0}$ (this solution is classical), see [2] for the proof. If, in addition to the growth condition (1.2), $f$ satisfies the dissipativeness condition (1.3) then all solutions $\mathbb{R} \ni t \mapsto u\left(t, u_{0}\right) \in$ $H_{0}^{1}\left(\Omega_{\epsilon}\right)$ of $(1.1)$ exist for all $t \geq 0$. Define the continuous nonlinear semigroup $\left\{T_{\epsilon}(t): H_{0}^{1}\left(\Omega_{\epsilon}\right) \rightarrow H_{0}^{1}\left(\Omega_{\epsilon}\right): t \geq 0\right\}$ by $\mathbb{R}^{+} \ni t \mapsto T_{\epsilon}(t) u_{0}:=u\left(t, u_{0}\right) \in H_{0}^{1}\left(\Omega_{\epsilon}\right)$, were $u\left(t, u_{0}\right) \in C\left([0, \infty), H_{0}^{1}\left(\Omega_{\epsilon}\right)\right) \cap C^{1}\left((0, \infty), C^{2}\left(\Omega_{\epsilon}\right)\right)$ is the classical solution to $(1.1)$.

If $f$ satisfies (1.2) and (1.3), it is shown in [3] that the problem (1.1) has a global attractor $\mathcal{A}_{\epsilon}$ and that

$$
\sup _{0 \leq \epsilon \leq \epsilon_{0}} \sup _{x \in \Omega_{\epsilon}} \sup _{u \in \mathcal{A}_{\epsilon}}|u(x)|<\infty .
$$

With this information in hand we can assume without loss of generality that $f$ is bounded in $C^{2}(\mathbb{R})$ ( $f$ can be cut without changing the attractor in such a way that it is $C^{2}(\mathbb{R})$-bounded). Hereafter we assume that $f, f^{\prime}$ and $f^{\prime \prime}$ are bounded.

Here we note that the attractor $\mathcal{A}_{\epsilon}$ is a subset of $H_{0}^{1}\left(\Omega_{\epsilon}\right)$. This is saying that as the parameter varies the space where $\mathcal{A}_{\epsilon}$ lives changes and in order to 
compare such sets we proceed in the following manner: Let $\mathcal{B}$ be a bounded smooth subset of $\mathbb{R}^{n}$ such that $\overline{U_{0 \leq \epsilon \leq \epsilon_{0}} \Omega_{\epsilon}} \subset \mathcal{B}$ and extend the solutions of (1.1) to $\mathcal{B}$ by zero outside $\Omega_{\epsilon}$ and consider them as functions in $H^{1}(\mathcal{B})$. With this understanding we may compare the sets $\mathcal{A}_{\epsilon}$. We say that the family of attractors $\left\{\mathcal{A}_{\epsilon}: 0 \leq \epsilon \leq \epsilon_{0}\right\}$ is upper semi-continuous at $\epsilon=0$ if $\lim _{\epsilon \rightarrow 0} \sup _{u \in \mathcal{A}_{\epsilon}} d\left(u, \mathcal{A}_{0}\right)=$ 0 (here $\left.d(a, \mathcal{C})=\inf _{c \in \mathcal{C}}\|a-c\|_{H^{1}(\mathcal{B})}\right)$ and we say that $\left\{\mathcal{A}_{\epsilon}: 0 \leq \epsilon \leq \epsilon_{0}\right\}$ is lower semi-continuous at $\epsilon=0$ if $\lim _{\epsilon \rightarrow 0} \sup _{u \in \mathcal{A}_{0}} d\left(u, \mathcal{A}_{\epsilon}\right)=0$.

In this paper we prove that the family of attractors $\left\{\mathcal{A}_{\epsilon}: 0 \leq \epsilon \leq \epsilon_{0}\right\}$ for (1.1) is upper and lower semi-continuous at $\epsilon=0$ in $H_{0}^{1}(\mathcal{B})$. The upper semi-continuity of attractors is, in general, a simple matter depending only on uniform bounds for the family of attractors (we will see that the bounds obtained in [3] and some uniform embedding results are enough to ensure upper semicontinuity), the lower semi-continuity is a much more delicate matter. A first and decisive task that must be addressed, to obtain the lower semi-continuity, is the continuity of the set of equilibria $\mathcal{E}_{\epsilon}$ for (1.1) (this is shown later in the paper). In order to be able to prove the continuity $\left\{\mathcal{E}_{\epsilon}: 0 \leq \epsilon \leq \epsilon_{0}\right\}$ we assume that for $\epsilon=0$ all the equilibria are hyperbolic (therefore only a finite number $m$ of equilibria exist) and prove that for small $\epsilon, \mathcal{E}_{\epsilon}$ has exactly $m$ equilibria and these converge to the equilibria for (1.1) with $\epsilon=0$. Once we have obtained the continuity of the set of equilibria we employ the gradient structure of (1.1) to obtain the lower semi-continuity of attractors using the results in $[7,8]$. This is done in the following manner: Once we have proved the continuity of the set of equilibria for (1.1), we have that the spectrum of the linearization around each equilibria behaves continuously with $\epsilon$ and as a consequence of the results in [7] we obtain the lower semicontinuity of the unstable manifold of each equilibrium. It follows from Theorem 4.10 .8 in [8] that the attractors $\mathcal{A}_{\epsilon}$ are lower-semicontinuous at $\epsilon=0$.

This paper is organized as follows: In Section 2 we study the upper semicontinuity of attractors proving some uniform embedding results, in Section 3 we prove that if $\mathcal{E}_{0}$ consists only of hyperbolic equilibria, then the family 
$\left\{\mathcal{E}_{\epsilon}, 0 \leq \epsilon \leq \epsilon_{0}\right\}$ is continuous at $\epsilon=0$.

\section{Upper semicontinuity of attractors}

In this section we will prove that the family of attractors $\mathcal{A}_{\epsilon}$ for (1.1) is upper semicontinuous at $\epsilon=0$. We start with the continuity of the spectrum for elliptic operators in such domains. The perturbations that we are allowing are well behaved in the following sense, if $\left(\lambda_{j, \epsilon}^{V_{\epsilon}}\right)_{\epsilon>0} \subset \mathbb{R} ;\left(\varphi_{j, \epsilon}^{V_{\epsilon}}\right)_{\epsilon>0} \subset H_{0}^{1}\left(\Omega_{\epsilon}\right), j \in \mathbb{N}$ are respectively the eigenvalues and eigenfunctions of the problem

$$
\left\{\begin{array}{cl}
-\Delta \varphi+V_{\epsilon} \varphi=\lambda \varphi & \text { in } \Omega_{\epsilon}, \\
\varphi=0 & \text { in } \partial \Omega_{\epsilon},
\end{array}\right.
$$

where $V_{\epsilon}: \mathbb{R}^{N} \rightarrow \mathbb{R}, 0 \leq \epsilon \leq \epsilon_{0}$, are such that $\left\|V_{\epsilon}\right\|_{L^{\infty}\left(\mathbb{R}^{N}\right)} \leq C, 0 \leq \epsilon \leq \epsilon_{0}$ and $V_{\epsilon} \stackrel{L^{2}}{\stackrel{\left(\mathbb{R}^{N}\right)}{\longrightarrow}} V_{0}$, then we must have that

$$
\lambda_{j, \epsilon}^{V_{\epsilon}} \longrightarrow \lambda_{j}^{V_{0}}, \varphi_{j, \epsilon}^{V_{\epsilon}} \longrightarrow \varphi_{j}^{V_{0}} \quad \text { as } \epsilon \longrightarrow 0 \quad \text { and } \forall j \in \mathbb{N}
$$

where $\left(\lambda_{j}^{V_{0}}\right)_{j \in \mathbb{N}} \subset \mathbb{R}$ and $\left(\varphi_{j}^{V_{0}}\right)_{j \in \mathbb{N}} \subset H_{0}^{1}\left(\Omega_{\epsilon}\right)$ are respectively the eigenvalues and eigenfunctions of the problem $(\lambda)_{0}$. We simply write $\lambda_{j, \epsilon}, \varphi_{j, \epsilon}$ for $\lambda_{j, \epsilon}^{0}, \varphi_{j, \epsilon}^{0}$ and $\lambda_{j}, \varphi_{j}$ for $\lambda_{j, 0}^{0}, \varphi_{j, 0}^{0}$. See $[1,4]$ and references therein.

A series of uniform bounds for attractors of parabolic problems is obtained in [3]. Next we state the results of [3] that will be used to obtain upper semicontinuity of attractors.

Proposition 2.1. Assume that (1.2) and (1.3) are satisfied. Then, for each $C_{0}>0$, there is a constant $C_{1}>0$ such that $u f(u) \leq C_{0} u^{2}+C_{1}|u|$. If $2 C_{0}<\lambda_{1}$ and we denote by $\phi$ the solution of

$$
\begin{array}{ll}
\Delta \phi_{\epsilon}+C_{0} \phi_{\epsilon}+C_{1}=0 & x \in \Omega_{\epsilon}, \\
\phi_{\epsilon}=0 & x \in \partial \Omega_{\epsilon},
\end{array}
$$

then $0 \leq \phi \in L^{\infty}\left(\Omega_{\epsilon}\right)$, $\sup _{0 \leq \epsilon \leq \epsilon_{0}}\left\|\phi_{\epsilon}\right\|_{L^{\infty}\left(\Omega_{\epsilon}\right)}<\infty, \lim _{t \rightarrow \infty}\left|u\left(t, x, u_{0}\right)\right| \leq \phi_{\epsilon}(x)$, uniformly in $x \in \bar{\Omega}_{\epsilon}$ and for $u_{0}$ in bounded subsets of $H_{0}^{1}\left(\Omega_{\epsilon}\right)$. In particular, we have that for any $v \in \mathcal{A}_{\epsilon}$ we have $|v(x)| \leq \phi_{\epsilon}(x), 0 \leq \epsilon \leq \epsilon_{0}$. 
and, if $H_{D}^{2}\left(\Omega_{\epsilon}\right)$ denotes the space $H^{2}\left(\Omega_{\epsilon}\right) \cap H_{0}^{1}\left(\Omega_{\epsilon}\right)$ with the norm

$$
\|u\|_{H_{D}^{2}\left(\Omega_{\epsilon}\right)}=\|\Delta u\|_{L^{2}\left(\Omega_{\epsilon}\right)}+\|u\|_{L^{2}\left(\Omega_{\epsilon}\right)}
$$

we have the following uniform embedding result

Theorem 2.1. Assume $f$ satisfies (1.2) and (1.3). Then, problem ((1.1)) has a global compact attractor, $\mathcal{A}_{\epsilon}$, in $H_{0}^{1}\left(\Omega_{\epsilon}\right)$ such that

$$
\begin{aligned}
& \text { (a) } \mathcal{A}_{\epsilon} \subset \Sigma\left(\phi_{\epsilon}\right):=\left\{u \in L^{\infty}\left(\Omega_{\epsilon}\right),|u(x)| \leq \phi_{\epsilon}(x) \text {, a.e. } x \in \Omega_{\epsilon}\right\} \\
& \text { (b) } \sup _{0 \leq \epsilon \leq \epsilon_{0}} \sup _{u_{0} \in \mathcal{A}_{\epsilon}} \sup _{t \in \mathbb{R}}\left\|u\left(t, u_{0}\right)\right\|_{H^{1}\left(\Omega_{\epsilon}\right)}<\infty . \\
& \text { (c) } \sup _{0 \leq \epsilon \leq \epsilon_{0}} \sup _{u_{0} \in \mathcal{A}_{\epsilon}} \sup _{t \in \mathbb{R}}\left\|u_{t}\left(t, u_{0}\right)\right\|_{H^{1}\left(\Omega_{\epsilon}\right)}<\infty . \\
& \text { (d) } \sup _{0 \leq \epsilon \leq \epsilon_{0}} \sup _{v \in \mathcal{A}_{\epsilon}}\|v\|_{H_{D}^{2}\left(\Omega_{\epsilon}\right)}<\infty .
\end{aligned}
$$

Before we proceed we prove the following uniform embedding result

Theorem 2.2. The embedding bellow are bounded uniformly for $0 \leq \epsilon \leq \epsilon_{0}$

$$
\begin{aligned}
& \text { 1. } E_{\epsilon}^{1,0}: H_{0}^{1}\left(\Omega_{\epsilon}\right) \hookrightarrow L^{\frac{2 N}{N-2}}\left(\Omega_{\epsilon}\right) \text {, } \\
& \text { 2. } E_{\epsilon, p}^{2,0}: H_{D}^{2}\left(\Omega_{\epsilon}\right) \hookrightarrow L^{p}\left(\Omega_{\epsilon}\right), 1 \leq p \leq \frac{2 N}{N-4} \text {, se } N>4 \text { and } \forall p \geq 1 \text {, se } N=4 \text {, } \\
& \text { 3. } E_{\epsilon, p}^{2,0}: H_{D}^{2}\left(\Omega_{\epsilon}\right) \hookrightarrow L^{\infty}\left(\Omega_{\epsilon}\right) \text {, se } N<4 \text {. }
\end{aligned}
$$

Furthermore, the embedding operator

$$
E_{\epsilon}^{2,1}: H_{D}^{2}\left(\Omega_{\epsilon}\right) \hookrightarrow H_{0}^{1}(\mathcal{B})
$$

is uniformly bounded $0 \leq \epsilon \leq \epsilon_{0}$ and if $u_{\epsilon} \in H_{D}^{2}\left(\Omega_{\epsilon}\right)$ is such that $\left\|u_{\epsilon}\right\|_{H_{D}^{2}\left(\Omega_{\epsilon}\right)} \leq 1$, $0 \leq \epsilon \leq \epsilon_{0}$, then $\left\{u_{\epsilon}, 0 \leq \epsilon \leq \epsilon_{0}\right\}$ is relatively compact in $H^{1}(\mathcal{B})$.

Proof: The proof of (1) follows immediately extending all functions to $B$ and using the embedding of $H^{1}(\mathcal{B})$ into $L^{\frac{2 N}{N-2}}(\mathcal{B})$. We prove (2) only in the case $N>4$ (the remaining case follows similarly). If $f \in L^{2}\left(\Omega_{\epsilon}\right)$ and

$$
\begin{gathered}
-\Delta u+u=f, \quad \text { in } \Omega_{\epsilon} \\
u=0, \quad \text { in } \partial \Omega_{\epsilon}
\end{gathered}
$$


we will prove that $u \in L^{\frac{2 N}{N-4}}\left(\Omega_{\epsilon}\right)$ and that there is a constant independent of $\epsilon$ and of $u, f$ such that $\|u\|_{L^{\frac{2 N}{N-4}}\left(\Omega_{\epsilon}\right)} \leq c\|f\|_{L^{2}\left(\Omega_{\epsilon}\right)}$. For that let $\phi=\max \{u, 0\}$, multiply (2.3) by $\phi^{r-1}$ and integrate by parts to obtain

$$
\begin{aligned}
4 \frac{r-1}{r^{2}} \int_{\Omega_{\epsilon}}\left|\nabla \phi^{\frac{r}{2}}\right|^{2}+\int_{\Omega_{\epsilon}}\left(\phi^{\frac{r}{2}}\right)^{2} & =\int_{\Omega_{\epsilon}} \Delta u \phi^{r-1}+\int_{\Omega_{\epsilon}} \phi^{r}=\int_{\Omega_{\epsilon}} f \phi^{r-1} \\
& \leq\|f\|_{L^{2}\left(\Omega_{\epsilon}\right)}\left\|\phi^{r-1}\right\|_{L^{2}\left(\Omega_{\epsilon}\right)} \\
& \leq\|f\|_{L^{2}\left(\Omega_{\epsilon}\right)}\left\|\phi^{\frac{r}{2}}\right\|_{L^{\frac{2}{r}}\left(\Omega_{\epsilon}\right)}
\end{aligned}
$$

making $\frac{4}{r^{\prime}}=\frac{2 N}{N-2}$ we have that $\frac{r}{2}=\frac{N-2}{N-4}$ and

$$
4 c \frac{r-1}{r^{2}}\left\|\phi^{\frac{r}{2}}\right\|_{L^{\frac{2 N}{N-2}}\left(\Omega_{\epsilon}\right)}^{2} \leq 4 \frac{r-1}{r^{2}} \int_{\Omega_{\epsilon}}\left|\nabla \phi^{\frac{r}{2}}\right|^{2}+\int_{\Omega_{\epsilon}}\left(\phi^{\frac{r}{2}}\right)^{2} \leq\|f\|_{L^{2}\left(\Omega_{\epsilon}\right)}\left\|\phi^{\frac{r}{2}}\right\|_{L^{\frac{2 N}{N-2}}\left(\Omega_{\epsilon}\right)}^{\frac{2}{r^{\prime}}}
$$

where we have used the embedding of $H^{1}\left(\Omega_{\epsilon}\right)$ into $L^{\frac{2 N}{N-2}}\left(\Omega_{\epsilon}\right)$. Finally, we have that

$$
c \frac{N(N-4)}{(N-2)^{2}}\|\phi\|_{L^{\frac{2 N}{N-4}\left(\Omega_{\epsilon}\right)}}=4 c \frac{r-1}{r^{2}}\left\|\phi^{\frac{r}{2}}\right\|_{L^{\frac{2 N}{N-2}}\left(\Omega_{\epsilon}\right)}^{\frac{2}{r}} \leq\|f\|_{L^{2}\left(\Omega_{\epsilon}\right)}
$$

which is the desired result.

To prove (3) we note that, for $q \geq p, L^{q}\left(\Omega_{\epsilon}\right)$ is embedded in $L^{p}\left(\Omega_{\epsilon}\right)$ and the embedding constant depends only on $p, q$ and $\left|\Omega_{\epsilon}\right|$. Let $k \geq 0, \phi=\max \{u-k, 0\}$ and $A_{k}=\left\{x \in \Omega_{\epsilon}: u(x)>k\right\}$. Multiplying the equation (2.3) by $\phi$ and integrating by parts we arrive at

$$
c\|\phi\|_{L^{\frac{2 N}{N-2}}\left(\Omega_{\epsilon}\right)}^{2} \leq \int_{\Omega_{\epsilon}}|\nabla \phi|^{2}+\int_{\Omega_{\epsilon}} \phi^{2}=\int_{\Omega_{\epsilon}} f \phi \leq\|f\|_{L^{2}\left(\Omega_{\epsilon}\right)}\|\phi\|_{L^{2}\left(\Omega_{\epsilon}\right)}
$$

for some $c>0$ independent of $\epsilon$. Note that

$$
\|\phi\|_{L^{q}\left(\Omega_{\epsilon}\right)}\left|A_{k}\right|^{-\frac{N+2}{2 N}+1-\frac{1}{q}} \leq\|\phi\|_{L^{\frac{2 N}{N-2}\left(\Omega_{\epsilon}\right)}} .
$$

From this, for $q=1$ and $q=2$, we have,

$$
c\|\phi\|_{L^{1}\left(\Omega_{\epsilon}\right)}^{2} \leq\|f\|_{L^{2}\left(\Omega_{\epsilon}\right)}\|\phi\|_{L^{\frac{2 N}{N-2}\left(\Omega_{\epsilon}\right)}}\left|A_{k}\right|^{1+\frac{2}{N}-\frac{1}{2}} .
$$

Since $\frac{2}{N}-\frac{1}{2}>0$ the result follows from Lemma 5.1 in [10], page 71 .

The embedding (2.2) is trivial from the identity

$$
\int_{\Omega_{\epsilon}}\left|\nabla u_{\epsilon}\right|^{2}=-\int_{\Omega_{\epsilon}} \Delta u_{\epsilon} u_{\epsilon}, \quad \forall u_{\epsilon} \in H_{D}^{2}\left(\Omega_{\epsilon}\right),
$$


from the embedding (2) and from Cauchy-Schwarz inequality. The compactness is proved in the following manner:

a) If $\tilde{u}_{\epsilon}$ denote the extension of $u_{\epsilon}$ to $\mathcal{B}$ by zero we have that there is a function $\tilde{u} \in H^{1}(\mathcal{B})$ and a sequence $\epsilon_{n} \rightarrow 0$ such that $\tilde{u}_{\epsilon_{n}} \rightarrow \tilde{u}$ weakly in $H^{1}(\mathcal{B})$, strongly in $L^{2}(\mathcal{B})$ and almost everywhere. It follows that $\tilde{u}=0$ almost everywhere in $B \backslash \Omega_{0}$ and therefore the trace of $\tilde{u}$ in $\partial \Omega_{0}$ is zero. Let $u$ be the restriction of $\tilde{u}$ to $\Omega_{0}$. The above considerations are saying that $u \in H_{0}^{1}\left(\Omega_{0}\right)$.

b) If

$$
f_{\epsilon_{n}}(x)=\left\{\begin{array}{l}
\Delta u_{\epsilon_{n}}(x) \quad x \in \Omega_{\epsilon_{n}} \\
0, \quad x \in B \backslash \Omega_{0}
\end{array}\right.
$$

we have that $\left\|f_{\epsilon_{n}}\right\|_{L^{2}(\mathcal{B})} \leq 1$, for all $n \geq 1$ and therefore it has a weakly convergent subsequence (which we again denote by $f_{\epsilon_{n}}$ ) to $f \in L^{2}(\mathcal{B})$. It is easy to see that $f=0$ almost everywhere in $\mathcal{B} \backslash \Omega_{0}$. From this we have that for every $\varphi \in C_{c}^{\infty}\left(\Omega_{0}\right)$ and sufficiently small $\epsilon$

$$
\int_{\mathcal{B}} u_{\epsilon_{n}} \Delta \varphi=\int_{\Omega_{\epsilon}} u_{\epsilon_{n}} \Delta \varphi=\int_{\Omega_{\epsilon_{n}}} \Delta u_{\epsilon} \varphi=\int_{\mathcal{B}} f_{\epsilon} \varphi
$$

taking the limit we obtain that

$$
\int_{\Omega_{0}} u \Delta \varphi=\int_{\Omega_{0}} f \varphi, \quad \forall \varphi \in C_{c}^{\infty}\left(\Omega_{0}\right)
$$

and $f=\Delta u \in L^{2}\left(\Omega_{0}\right)$. Hence, $u \in H_{D}^{2}\left(\Omega_{0}\right)$.

Thus

$$
\left\|\nabla \tilde{u}_{\epsilon_{n}}\right\|_{L^{2}(\mathcal{B})}^{2}=\left\|\nabla u_{\epsilon_{n}}\right\|_{L^{2}\left(\Omega_{\epsilon_{n}}\right)}^{2}=\left\langle\Delta u_{\epsilon_{n}}, u_{\epsilon_{n}}\right\rangle_{L^{2}\left(\Omega_{\epsilon_{n}}\right)} \rightarrow\langle f, u\rangle_{L^{2}\left(\Omega_{0}\right)}=\int_{\mathcal{B}}|\nabla \tilde{u}|^{2} .
$$

This proves that $\tilde{u}_{\epsilon_{n}} \rightarrow \tilde{u}$ strongly in $H^{1}(\mathcal{B})$ and we have the result.

With the uniform bounds given in Theorem 2.1 we obtain the following result

Theorem 2.3. The problem (1.1), for $0<\epsilon \leq \epsilon_{0}$, has an attractor $\mathcal{A}_{\epsilon}$ and the family $\left\{\mathcal{A}_{\epsilon}: 0<\epsilon \leq \epsilon_{0}\right\}$ is uniformly bounded in $H^{1}(\mathcal{B})$ (recall that the functions of $H_{0}^{1}\left(\Omega_{\epsilon}\right)$ are extended by zero outside $\left.\Omega_{\epsilon}\right)$. Then the family $\left\{\mathcal{A}_{\epsilon}: 0 \leq \epsilon \leq \epsilon_{0}\right\}$ is upper semi-continuous at $\epsilon=0$ in $H^{1}(\mathcal{B})$. 
Prova: It follows from the results in [3] that the problems (1.1) have global attractors $\mathcal{A}_{\epsilon}, 0<\epsilon \leq \epsilon_{0}$, which are uniformly bounded in $H^{1}(\mathcal{B})$.

Now, for each $\epsilon$ take a global trajectory $u^{\epsilon}(t)$ lying on the attractor $\mathcal{A}_{\epsilon}$.

From the results in [3] we obtain that

$$
\left\|\nabla u^{\epsilon}(t)\right\|_{L^{2}(\mathcal{B})},\left\|\nabla u_{t}^{\epsilon}(t)\right\|_{L^{2}(\mathcal{B})},\left\|\Delta u_{\epsilon}\right\|_{L^{2}\left(\Omega_{\epsilon}\right)}
$$

are uniformly bounded for $0 \leq \epsilon \leq \epsilon_{0}$ and $t \in \mathbb{R}$.

This implies that for fixed $t, u^{\epsilon}(t)$ is relatively compact in $H^{1}(\mathcal{B})$.

Hence, by Arzelá-Ascoli Theorem (taking subsequences if necessary), we have that

$$
u^{\epsilon} \rightarrow u \quad \text { locally uniformly in } C\left(\mathbb{R}, H^{1}(\mathcal{B})\right) \text {. }
$$

Following an standard argument we consider the weak formulation of (1.1),

$$
\int_{\Omega_{\epsilon}} u_{t}^{\epsilon} \varphi=-\int_{\Omega_{\epsilon}} \nabla u^{\epsilon} \nabla \varphi+\int_{\Omega_{\epsilon}} f\left(u^{\epsilon}\right) \varphi
$$

for all smooth test functions $\varphi$. For each test function $\phi \in C_{0}^{\infty}\left(\Omega_{0}\right)$, with support $K$, there is a constant $\epsilon_{K}$ such that $K \subset \subset \Omega_{\epsilon}, 0 \leq \epsilon \leq \epsilon_{K}$ and the integrals in the above identity happens in $\Omega_{0}$.

From the convergence of $u^{\epsilon}$, we can pass to the limit and obtain that the limiting function $u$ satisfies

$$
\int_{\Omega_{0}} u_{t} \varphi=-\int_{\Omega_{0}} \nabla u \nabla \varphi+\int_{\Omega_{0}} f(u) \varphi
$$

for each $\phi \in C_{0}^{\infty}\left(\Omega_{0}\right)$. Thus, $u$ is a globally defined solution to (1.1), with $\epsilon=0$, which is bounded in $H^{1}\left(\Omega_{0}\right) \cap C\left(\bar{\Omega}_{0}\right)$. Hence $u(t) \in \mathcal{A}_{0}$ for all $t \in \mathbb{R}$.

The rest of the proof now follows in an standard way (see, for example, [9]).

\section{Continuity of the set of Equilibria}

Let $\Omega_{0} \subset \mathbb{R}^{N}(N \leq 3)$ be a bounded smooth domain and $f: \mathbb{R} \longrightarrow \mathbb{R}$ be a $C^{2}(\mathbb{R})$ function with

$$
|f(u)| \leq c_{f}, \quad\left|f^{\prime}(u)\right| \leq c_{f}^{\prime}, \quad\left|f^{\prime \prime}(u)\right| \leq c_{f}^{\prime \prime} \quad \forall u \in \mathbb{R}
$$


where $c_{f}$ and $c_{f}^{\prime}$ are positive constants.

In this section we will address the following problem

$$
\begin{cases}\Delta u+f(u)=0 & \text { in } \Omega_{\epsilon} \\ u=0 & \text { in } \partial \Omega_{\epsilon} .\end{cases}
$$

for each $0 \leq \epsilon \leq \epsilon_{0}$.

Assume that the problem $(P)_{0}$ has exactly $m$ distinct solutions, $u_{1}, \ldots, u_{m}$ and that zero is not an eigenvalue of the operator $\Delta+f^{\prime}\left(u_{i}\right) I$ for $i=1, \ldots, m$. Our goal is to show that, for small perturbations of the domain $\Omega_{0}$, there are yet exactly $m$ distinct solutions for the perturbed problem and, when the perturbed domains $\Omega_{\epsilon}$ converge (in the sense explained in the introduction) to the domain $\Omega_{0}$, they converge to the solutions of the problem $(P)_{0}$.

Once we have shown that the problem $(P)_{\epsilon}$ has exactly $m$ solutions $u_{1, \epsilon}, \ldots, u_{m, \epsilon}$, we show that $u_{i, \epsilon} \longrightarrow u_{i}$ strongly in $H^{1}(\mathcal{B}), i=1, \ldots, m$. Here, the functions $u_{k}$, $k=1, \ldots, m$ denote the $m$ solutions of the problem $(P)_{0}$.

The idea is to show that there are compact continuous maps $\mathcal{F}_{k}$ and $\mathcal{F}_{k, \epsilon}$, associated respectively to the problems $(P)_{0}$ and $(P)_{\epsilon}$. Then we look for fixed points of these operatores and show that for sufficiently small $\epsilon$ we have exactly $m$ solutions for the problem $(P)_{\epsilon}$ and that $\mathcal{F}_{k, \epsilon}\left(w_{k, \epsilon}\right)=w_{k, \epsilon} \longrightarrow \mathcal{F}\left(u_{k}\right)=u_{k}$, $\forall k=1, \ldots, m$.

It follows from the convergence of eigenvalues that, if $\lambda=0$ is in the resolvent set of the operator $\left(-\Delta+V_{0} I\right)$, then there exists $\bar{C}>0$ such that

$$
\limsup _{\epsilon \rightarrow 0}\left\|\left(-\Delta+V_{\epsilon} I\right)^{-1}\right\|_{L\left(L^{2}\left(\Omega_{\epsilon}\right), L^{2}\left(\Omega_{\epsilon}\right)\right)} \leq \bar{C} .
$$

From this, we obtain that

$$
\limsup _{\epsilon \rightarrow 0}\left\|\left(-\Delta+V_{\epsilon} I\right)^{-1}\right\|_{L\left(L^{2}\left(\Omega_{\epsilon}\right), H^{1}\left(\Omega_{\epsilon}\right)\right)} \leq C,
$$

for some $C>0$. To prove this let $f \in L^{2}\left(\Omega_{\epsilon}\right)$ and $u \in H_{D}^{2}\left(\Omega_{\epsilon}\right)$ be such that

$$
-\Delta u+V_{\epsilon} u=f .
$$


Multiplying this equation by $u$ and integrating by parts we obtain that

$$
\|\nabla u\|_{L^{2}\left(\Omega_{\epsilon}\right)}^{2} \leq \bar{C}\left(\bar{C}\left\|V_{\epsilon}\right\|_{L^{\infty}\left(\Omega_{\epsilon}\right)}+1\right)\|f\|_{L^{2}\left(\Omega_{\epsilon}\right)}^{2}
$$

and the result follows.

Next we define de operators $\mathcal{F}_{\epsilon}, \mathcal{F}_{k, \epsilon}$ and verify some facts about them. Let $\left(\mathcal{F}_{\epsilon}\right)_{\epsilon \geq 0}$, where $\mathcal{F}_{0}=\mathcal{F}$, simply putting

$$
\begin{aligned}
\mathcal{F}_{\epsilon}: H_{0}^{1}\left(\Omega_{\epsilon}\right) & \longrightarrow H_{0}^{1}\left(\Omega_{\epsilon}\right) \\
v_{\epsilon} & \mapsto \mathcal{F}_{\epsilon}\left(v_{\epsilon}\right)
\end{aligned}
$$

in such a way that

$(P)_{o, \epsilon}$

$$
\left\{\begin{array}{cl}
-\Delta \mathcal{F}_{\epsilon}\left(v_{\epsilon}\right)=f\left(v_{\epsilon}\right) & \text { in } \Omega_{\epsilon}, \\
\mathcal{F}_{\epsilon}\left(v_{\epsilon}\right)=0 & \text { in } \partial \Omega_{\epsilon} .
\end{array}\right.
$$

Since this problem has a unique solution, see [[5]-Cap. X], the operator $\mathcal{F}_{\epsilon}$ is well defined.

Proposition 3.1. The fixed points of $\mathcal{F}_{\epsilon}$, converge to the fixed points of $\mathcal{F}_{0}$.

Proof: In fact, suppose that $\left(v_{\epsilon}\right)_{\epsilon>0} \subset H_{0}^{1}\left(\Omega_{\epsilon}\right)$, for each $\epsilon>0$ is a fixed point of $\mathcal{F}_{\epsilon}$. So

$$
\int_{\Omega_{\epsilon}} \nabla v_{\epsilon} \nabla \varphi_{\epsilon}=\int_{\Omega_{\epsilon}} f\left(v_{\epsilon}\right) \varphi_{\epsilon}, \quad \forall \varphi_{\epsilon} \in H_{0}^{1}\left(\Omega_{\epsilon}\right) .
$$

From the hypothesis $f(u) u<0$ for $|u| \geq R$ and for some $R>0$, we conclude that the sequence $v_{\epsilon}$ is uniformly bounded in $H_{0}^{1}\left(\Omega_{\epsilon}\right)$. From this we conclude that there is a sequence $\epsilon_{n} \rightarrow 0$ such that $\left(v_{\epsilon_{n}}\right)$ (viewed as a sequence in $H^{1}(\mathcal{B})$ ) converges to $v \in H^{1}(\mathcal{B})$ weakly in $H^{1}(\mathcal{B})$ and strongly in $L^{2}(\mathcal{B})$. Since $v=0$ almost everywhere in $B \backslash \Omega_{0}$ we have that $v \in H_{0}^{1}\left(\Omega_{0}\right)$.

From the hypotheses on $\Omega_{\epsilon}$, for each $K \subset \subset \Omega_{0}$, there is $\epsilon_{K}$ such that, $K \subset \subset \Omega_{\epsilon} 0 \leq \epsilon \leq \epsilon_{K}$. Then, if $\phi \in C_{0}^{\infty}\left(\Omega_{0}\right)$ we have that $\phi \in C_{0}^{\infty}\left(\Omega_{\epsilon}\right)$ $0 \leq \epsilon \leq \epsilon_{K}, K=\operatorname{supp}(\phi)$. Thus, for $n$ large,

$$
\int_{\Omega_{0}} \nabla v_{\epsilon_{n}} \nabla \phi=\int_{\Omega_{0}} f\left(v_{\epsilon_{n}}\right) \phi .
$$


Taking the limit as $n \rightarrow \infty$ we have that

$$
\int_{\Omega_{0}} \nabla v \nabla \phi=\int_{\Omega_{0}} f(v) \phi .
$$

The above argument holds for each compact $K \subset \subset \Omega_{0}$, and we have

$$
\int_{\Omega_{0}} \nabla v \nabla \phi=\int_{\Omega_{0}} f(v) \phi \quad \forall \phi \in H_{0}^{1}\left(\Omega_{0}\right) .
$$

Since $v \in H_{0}^{1}\left(\Omega_{0}\right)$ we have that $v=\mathcal{F}_{0}(v)$.

Since $f$ is bounded we have that $\left\{\left\|v_{\epsilon_{n}}\right\|_{H_{D}^{2}\left(\Omega_{\epsilon_{n}}\right)}: n \geq 1\right\}$ is a bounded sequence. It follows from Theorem 2.2 that $\left\{v_{\epsilon_{n}}\right\}$ converges strongly in $H^{1}(\mathcal{B})$ to $v$.

We know that $\mathcal{F}_{0}$ has exactly $m$ fixed points. Let us show that, for sufficiently small $\epsilon,\left(\mathcal{F}_{\epsilon}\right)_{\epsilon>0}$ has at least $m$ fixed points.

Proposition 3.2. The maps $\mathcal{F}_{\epsilon}$ defined in $(P)_{0, \epsilon}$ have at least $m$ fixed points in $H_{0}^{1}\left(\Omega_{\epsilon}\right)$ for sufficiently small $\epsilon>0$.

Proof: Note that, proceeding as before we can prove that each sequence of solutions to $(P)_{0, \epsilon}$ converges to a solution of $(P)_{0}$.

Now, since the solutions $u_{1}, \ldots, u_{m}$ of $(P)_{0}$ are distinct, we can chose $\delta>0$, such that the balls $B_{\delta}\left(u_{k}\right) \subset H^{1}\left(\Omega_{0}\right), k=1, \ldots, m$; are pairwise disjoint. Define the function $u_{k, \epsilon} k=1, \ldots, m$, as the unique solution of the problem

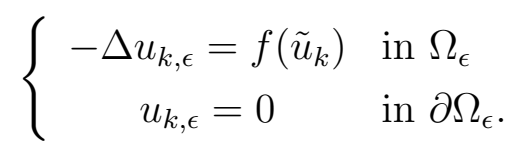

Here, $\tilde{u}_{k}$ represents the extension by zero outiside $\Omega_{0}$. Proceeding as in Proposition 3.1 , we can show that $u_{k, \epsilon} \longrightarrow u_{k}$ as $\epsilon \longrightarrow 0$ strongly in $H^{1}(\mathcal{B})$. Hence, for small $\epsilon>0$, we still have $B_{\delta}\left(u_{i, \epsilon}\right) \bigcap B_{\delta}\left(u_{j, \epsilon}\right)=\emptyset$, for $i, j=1, \ldots, m$ e $i \neq j$ (balls in $H_{0}^{1}\left(\Omega_{\epsilon}\right)$ ). Also note that $B_{\delta}\left(u_{k, \epsilon}\right) \subset H_{0}^{1}\left(\Omega_{\epsilon}\right)$, for $k=1, \ldots, m$.

To obtain the existence of solutions for the problem $(P)_{0, \epsilon}$, let us define the following auxiliary map

$$
\mathcal{F}_{k, \epsilon}\left(u_{\epsilon}\right)=\left(-\Delta+f^{\prime}\left(u_{k, \epsilon}\right)\right)^{-1}\left(f\left(u_{\epsilon}\right)+f^{\prime}\left(u_{k, \epsilon}\right) u_{\epsilon}\right) .
$$


for $k=1, \ldots, m, u_{\epsilon} \in H_{0}^{1}\left(\Omega_{\epsilon}\right)$. Note that $\mathcal{F}_{k, \epsilon}$ is continuous and compact for each $k=1, \ldots, m$.

To conclude we will use the following lemma

Lemma 3.1. $\mathcal{F}_{k, \epsilon}$ is a strict contraction from $B_{\delta}\left(u_{k, \epsilon}\right)$ into itself; that is, $\mathcal{F}_{k, \epsilon}\left(B_{\delta}\left(u_{k, \epsilon}\right)\right) \subset B_{\delta}\left(u_{k, \epsilon}\right)$ and $\mathcal{F}_{k, \epsilon}$ is a contraction.

Proof: Given $v_{\epsilon}, w_{\epsilon} \in \overline{B_{\delta}\left(u_{k, \epsilon}\right)}$, we have that:

$$
\left\|\mathcal{F}_{k, \epsilon}\left(v_{\epsilon}\right)-\mathcal{F}_{k, \epsilon}\left(w_{\epsilon}\right)\right\|_{H_{0}^{1}\left(\Omega_{\epsilon}\right)} \leq\left\|\mathcal{T}_{k, \epsilon}\right\|_{\mathcal{L}}\left\|f\left(v_{\epsilon}\right)-f\left(v_{\epsilon}\right)-f^{\prime}\left(u_{k, \epsilon}\right)\left(v_{\epsilon}-w_{\epsilon}\right)\right\|_{L^{2}\left(\Omega_{\epsilon}\right)}
$$

where $\mathcal{T}_{k, \epsilon}=\left(-\Delta-f^{\prime}\left(u_{k, \epsilon}\right)\right)^{-1}$ and $\mathcal{L}=L\left(L^{2}\left(\Omega_{\epsilon}, H^{1}\left(\Omega_{\epsilon}\right)\right.\right.$. Using

$$
f\left(v_{\epsilon}\right)-f\left(w_{\epsilon}\right)-f^{\prime}\left(u_{k, \epsilon}\right)\left(v_{\epsilon}-w_{\epsilon}\right)=\mathcal{R}_{w_{k, \epsilon}},
$$

where, from the fact that $f^{\prime \prime}$ is bounded, $\left|\mathcal{R}_{w_{k, \epsilon}}\right| \leq c\left(\left|v_{\epsilon}-u_{k, \epsilon}\right|+\left|w_{\epsilon}-u_{k, \epsilon}\right|\right)\left(v_{\epsilon}-\right.$ $\left.w_{\epsilon}\right)$. Hence

$$
\begin{aligned}
\int_{\Omega_{\epsilon}}\left|\mathcal{R}_{w_{k, \epsilon}}\right|^{2} & \leq c \int_{\Omega_{\epsilon}}\left(\left|v_{\epsilon}-u_{k, \epsilon}\right|^{2}+\left|w_{\epsilon}-u_{k, \epsilon}\right|^{2}\right)\left|v_{\epsilon}-w_{\epsilon}\right|^{2} \\
& \leq c\left[\left(\int_{\Omega_{\epsilon}}\left|v_{\epsilon}-u_{k, \epsilon}\right|^{N}\right)^{2}+\left(\int_{\Omega_{\epsilon}}\left|w_{\epsilon}-u_{k, \epsilon}\right|^{N}\right)^{2}\right]\left(\int_{\Omega_{\epsilon}}\left|v_{\epsilon}-w_{\epsilon}\right|^{\frac{2 N}{N-2}}\right)^{2} .
\end{aligned}
$$

Using Theorem 2.2, (1) we have that $\left\|\mathcal{R}_{w_{k, \epsilon}}\left(v_{\epsilon}-w_{\epsilon}\right)\right\|_{L^{2}\left(\Omega_{\epsilon}\right)} \leq \tilde{c} \delta\left\|v_{\epsilon}-w_{\epsilon}\right\|_{H^{1}\left(\Omega_{\epsilon}\right)}$, for $N \leq 4$. So,

$$
\left\|\mathcal{F}_{k, \epsilon}\left(v_{\epsilon}\right)-\mathcal{F}_{k, \epsilon}\left(w_{\epsilon}\right)\right\|_{H_{0}^{1}\left(\Omega_{\epsilon}\right)} \leq \tilde{c} \delta\left\|\mathcal{T}_{k, \epsilon}\right\|_{\mathcal{L}}\left\|v_{\epsilon}-w_{\epsilon}\right\|_{H^{1}\left(\Omega_{\epsilon}\right)}
$$

Note that, from (3.2),

$$
\left\|\mathcal{T}_{k, \epsilon}\right\|_{\mathcal{L}} \leq C
$$

with $C$ independent of $\epsilon$. With this, for sufficiently small $\delta>0$, we obtain that

$$
\tilde{c} C \delta<1
$$

Hence, $\mathcal{F}_{k, \epsilon}$ is a contraction. 
Conclusion of the Proof of Proposition 3.2 Now, in view of Lemma 3.1, we can apply the Banach Contraction Principle to the operator $\mathcal{F}_{k, \epsilon}$ in the closed set $\overline{B_{\delta}\left(u_{k, \epsilon}\right)}$ to obtain a fixed point $u_{\epsilon} \in B_{\delta}\left(u_{k, \epsilon}\right)$. With this we conclude the existence of at least $m$ solutions to the problem $(P)_{0, \epsilon}$.

In what follows we prove that, for small enough $\epsilon$, there are exactly $m$ equilibrium points for (1.1); that is, $\mathcal{F}_{k, \epsilon}$ has exactly $m$ fixed points. This is to say that the perturbed problem $(P)_{0, \epsilon}$ has exactly $m$ solutions for sufficiently small $\epsilon>0$.

We start with the following Corollary of the Proposition 3.2.

Corollary 3.1. The operator $\mathcal{A}_{k, \epsilon}$ has a unique fixed point in the ball $\overline{B_{\delta}\left(u_{k, \epsilon}\right)}$.

We know that each sequence of solutions of the problems $(P)_{0, \epsilon}$ converge, as $\epsilon \rightarrow 0$, to a solution of the problem $(P)_{0}$ (See Proposition 3.1). In particular, fixed points of $\mathcal{F}_{\epsilon}$ converge to fixed points of $\mathcal{F}_{0}$. Hence, recalling that there exists a unique fixed point satisfying

$$
\left\|u_{\epsilon}-u_{k, \epsilon}\right\|_{H_{0}^{1}\left(\Omega_{\epsilon}\right)} \leq \delta
$$

for $\epsilon>0$ small and for all $k$, we conclude that

Proposition 3.3. The fixed points $u_{\epsilon}$ of $\mathcal{F}_{\epsilon}$ that are in the neighborhood of $u_{k, \epsilon}$; that is, $u_{\epsilon} \in \overline{B_{\delta}\left(u_{k, \epsilon}\right)}$ are, for suitably small $\epsilon$, the only fixed points of $\mathcal{A}_{k, \epsilon}$.

Proof: Let $\epsilon_{1}$ be such that $\mathcal{F}_{k, \epsilon}$ has a unique fixed point in $B_{\delta}\left(u_{k, \epsilon}\right), 0 \leq \epsilon \leq \epsilon_{1}$, $1 \leq k \leq m$. Assume that there is a sequence $\epsilon_{n}$ such that $u_{\epsilon_{n}}$ is a fixed point of $\mathcal{F}_{k, \epsilon_{n}}$ such that $\left\|u_{\epsilon_{n}}-u_{k, \epsilon_{n}}\right\|_{L^{2}\left(\Omega_{\epsilon_{n}}\right)}>\delta, n=1,2,3 \cdots$.

From Proposition 3.1 there is $u \in H_{0}^{1}\left(\Omega_{0}\right)$ such that $u_{\epsilon_{n}} \longrightarrow u$ strongly in $H^{1}(\mathcal{B})$ as $n \rightarrow \infty$ and that $u \equiv u_{k}$ for some $1 \leq k \leq m$. From this we have that

$$
\limsup _{\epsilon \rightarrow 0}\left\|u_{\epsilon_{n}}-u_{k, \epsilon_{n}}\right\|_{H^{1}\left(\Omega_{\epsilon}\right)} \leq \lim _{\epsilon \rightarrow 0}\left\{\left\|u_{\epsilon_{n}}-u_{k}\right\|_{H^{1}(\mathcal{B})}+\left\|u_{k}-u_{k, \epsilon_{n}}\right\|_{H^{1}(\mathcal{B})}\right\}=0 .
$$

Thus, $u_{\epsilon_{n}} \in \overline{B_{\delta}\left(u_{k, \epsilon_{n}}\right)}$ for suitably large $n$ which is a contradiction and proves the result. 
This last result is telling us that for suitably small $\epsilon>0$, there are no

fixed points of the operator $\mathcal{F}_{k, \epsilon}$ outside the ball $\overline{B_{\delta}\left(u_{k, \epsilon}\right)}$ for if that was not the case a fixed point outside such balls would eventually enter these balls and contradicting the uniqueness of fixed points in these balls. Here the essential feature is the independence of $\delta$ relatively to $\epsilon$. With this, we have that the problem $(P)_{0, \epsilon}$ has precisely $m$ solution for small $\epsilon>0$.

Acknowledgement. This work was carried out while the first author visited Instituto de Ciências Matemáticas e de Computação at Universidade de São Paulo in São Carlos, Brazil. He wishes to acknowledge the hospitality of the people from this Institution.

\section{References}

[1] Arrieta, J. M., Spectral Properties of Schrödinger Operators Under Perturbations of the Domain, PhD Thesis, Georgia Institute of Technology, Atlanta GA, USA (1991).

[2] Arrieta, J. M.; Carvalho, A. N., Abstract parabolic problems with critical nonlinearities and applications to Navier-Stokes and heat equations, Trans. Amer. Math. Soc., 352(1) (2000), 285-310.

[3] Arrieta, J. M.; Carvalho, A. N.; Rodriguez-Bernal, A., Attractors for Parabolic Problems with Nonlinear Boundary Condition. Uniform Bounds, Communications in Partial Differential Equations 25, 1-2, (2000), 1-37.

[4] Babuska, I.; Vyborny, R., Continuous Dependence of Eigenvalues on the Domains, Czech. Math. J., 15 (1995) 169-178.

[5] Brézis, H., Analyse fonctionnelle, Théorie et applications, Masson, Paris, (1983).

[6] Gilbarg, D.; Trundiger, N. S., Elliptic Partial Differential Equations of Second Order, Grund. Math. Wiss. 224, Springer Verlag, Berlin (1983). 
[7] Carvalho, A. N.; Hines, G., Lower Semicontinuity of Unstable Manifolds for Gradient Systems, Dynamic Systems and Applications, Vol. 9, $\mathrm{n}^{\circ}$ 1, (2000), 37-50.

[8] Hale, J. K., Asymptotic Behavior of Dissipative Systems, Mathematical Surveys and Monographs, 25, AMS (1988).

[9] Hale, J. K.; Raugel, R., Upper semi-continuity of the attractor for a singularly perturbed hyperbolic equation, Journal of Diff. Equations 73, (1988). $197-214$.

[10] Ladyzenskaya, O.; Uralseva, N., Linear and Quasilinear Elliptic Equations, Academic Press (1968).

Departamento de Matemática-ICEx Universidade Federal de Minas Gerais Caixa Postal 702

30123-970, Belo Horizonte, MG

Brazil

E-mail:emerson@mat.ufmg.br
Departamento de Matemática-ICMC Universidade de São Paulo Caixa Postal 668 13560-970, São Carlos, SP Brazil

E-mail: andcarva@icmsc.sc.usp.br 